\title{
Ultra-smooth lithium niobate single crystal photonic micro-structures
}

\author{
S. Mailis ${ }^{1}$, Y. J. Ying ${ }^{1}$, C. L. Sones ${ }^{1}$, A. C. Peacock ${ }^{1}$, F. Johann ${ }^{2}$, E. Soergel ${ }^{2}$, R. W. Eason ${ }^{1}$, and M. N. Zervas ${ }^{1}$ \\ ${ }^{1}$ Optoelectronics Research Centre, University of Southampton, Highfield, Southampton, SO17 1BJ, Southampton, UK \\ ${ }^{2}$ Institute of Physics, University of Bonn, Wegelerstrasse 8, 53115 Bonn, Germany \\ sm@orc.soton.ac.uk
}

\begin{abstract}
Thermal treatment of micro-structured lithium niobate, at temperatures close to the Curie point, induces preferential surface melting and re-crystallization. This process yields ultrasmooth single crystal superstructures suitable for fabrication of low scattering loss photonic microcomponents.

(C)2009 Optical Society of America

OCIS codes: (220.0220) Optical design and fabrication; (230.0230) Optical devices
\end{abstract}

1. Introduction. One of the sources of optical power loss and performance degradation in photonic devices, such as whispering gallery mode (WGM) micro-resonators, is surface imperfections and/or sharp edges which induce scattering due to the dielectric contrast between the device and the surrounding material. These fabricationimperfections can be corrected by thermal treatment i.e. using a $\mathrm{CO}_{2}$ laser to locally melt the surface allowing for the surface tension to reshape the relevant structure to form a perfect extremely smooth globule. WGM cavities which are fabricated in this way exhibit very low scattering loss and hence very high $\mathrm{Q}$ factors [1,2].

Although such a method can be applied to glass without a problem, it is rather different if applied to single crystal materials mainly because the single crystal nature of the material is not preserved after a rapid melting and cooling cycle [3]. The resulting poly-crystalline material, which will suffer from light being scattered at the boundaries between adjacent crystallites, is therefore not suitable for electro-optic and non-linear applications.

Here we present a method where thermal treatment at temperatures close to the Curie point is seen to induce preferential surface melting of micron-sized superstructures in single crystal lithium niobate. Upon cooling the melt re-crystallizes, seeded by the bulk that remains solid during the process, and is reshaped by the surface tension to form ultra smooth single crystal superstructures.

2. Thermal treatment. Figure 1a shows a scanning electron microscopy (SEM) image of the micro-structured surface of a lithium niobate sample before thermal treatment. The surface consists of a 2D lattice of micro-pyramids; a result of deep etching following ferroelectric domain inversion [4]. This sample was chosen due to its unusually rough side surface of the pyramids to illustrate the improvement of the surface quality due to melting and subsequent re-growth. The crystal was then thermally treated with the temperature being raised to $1130^{\circ} \mathrm{C}$ for several hours followed by slow cooling back to room temperature. The heat treatment was performed in an oxygen atmosphere to suppress Li out-diffusion.
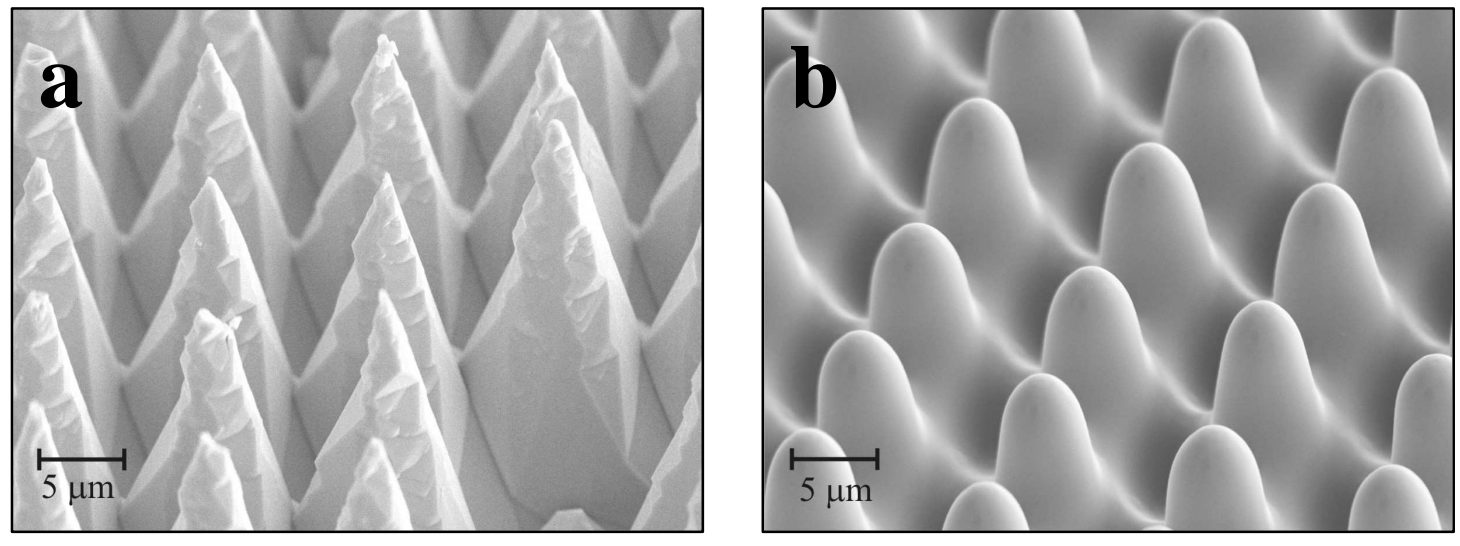

Fig1. SEM images of the micro-structured lithium niobate crystal surface a) after deep etching of a 2D domain lattice and b) after thermal treatment.

The post-treatment surface of the sample is shown in the SEM image of Fig. 1b where a dramatic change of the surface topography can be observed. Although the original 2D periodic micro-pyramid lattice is still visible, the structures appear rounded and the initially rough side surfaces have become remarkably smooth. The resulting 
structure (shown in Fig. 1b) is a smoother version of the initial one shown in Fig. 1a, hence it seems possible to define the final shape by choosing the initial structure.
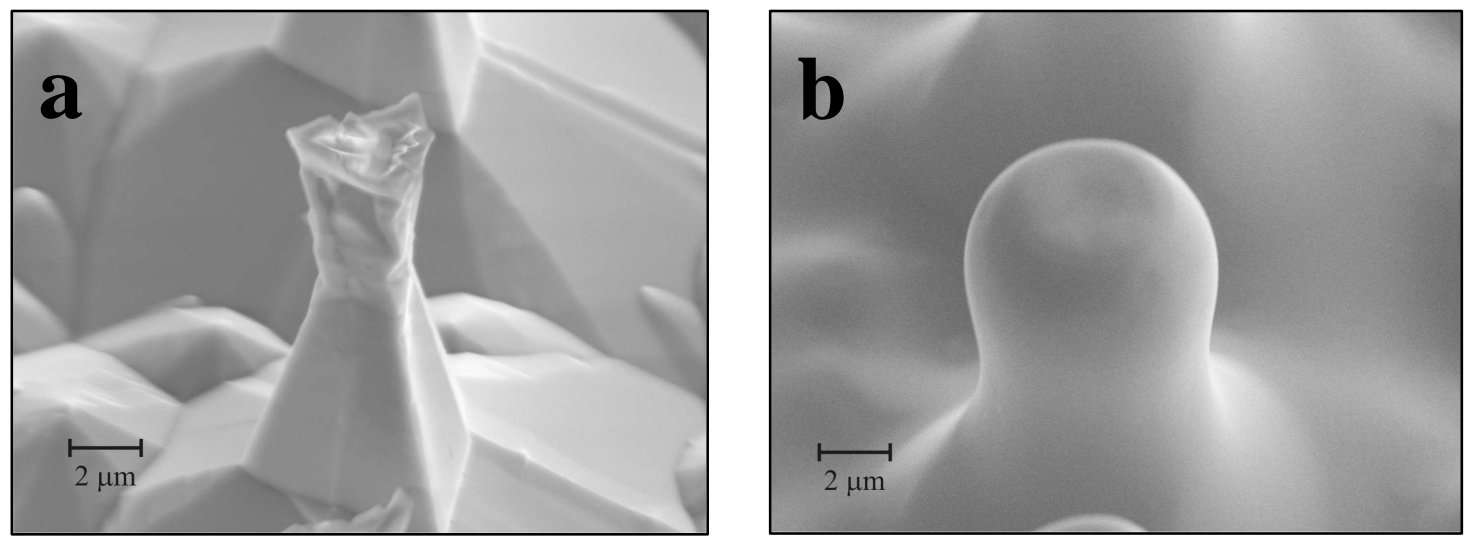

Fig. 2 a) Initial structure with an undercut produced by inhibition of poling followed by deep chemical etching using HF acid, b) corresponding annealed structure showing an oblate spheroid top.

Fig. 2a shows an undercut etched structure which has been produced by deep etching of a poling-inhibited surface ferroelectric domain structure [5]. Observation of the post-treatment surface, shown in the SEM image of Fig. $2 b$, confirms that the memory of the undercut has been preserved in the annealed structure thus producing a structure with an oblate spheroid top, which is a suitable geometry to form a WGM micro-resonator.

3. Discussion. Although the range of temperatures that the lithium niobate crystals experienced during the thermal treatment were kept below the Curie point $\left(1142^{\circ} \mathrm{C}\right)$ and hence well below the melting point $\left(1257^{\circ} \mathrm{C}\right)$ it is evident that, at some point during the process, the surface was in the liquid phase in both examples shown in Figs. $1 \mathrm{~b}$ and $2 b$. However, preferential melting of the surface is possible at temperatures lower than the bulk melting temperature, with the thickness of the melted zone being a function of the temperature [6].

Since the temperature was kept below the Curie point it is expected that the bulk of the processed material is still single crystal and ferroelectric throughout the thermal processing, however this is not necessarily true for the surface. Investigation of the annealed crystal surface using piezoresponse force microscopy (PFM) showed that the melted and re-solidified surface is also piezoelectric with a piezoresponse identical to the bulk, thus the re-solidified material is also both single crystal and ferroelectric. The single crystal nature of the surface was also investigated by micro-Raman spectroscopy and chemical etching, both of which verified the PFM result.

The collective results regarding the crystal quality at the surface after thermal treatment suggest that the melted layer re-crystallizes upon cooling, seeded by the solid crystal underneath, so that it follows the same crystallographic orientation as the substrate.

4. Conclusions. We have demonstrated a method for achieving ultra-smooth single crystal lithium niobate structures enabling the fabrication of low scattering loss photonic structures. The method is based on selective surface melting at temperatures below the Curie point, followed by seeded re-crystallization.

5. Acknowledgements. The authors would like to thank the E.U. and the EPSRC for funding via the STREP 3D - DEMO and EP/C515668/1 portfolio grant respectively.

\section{References.}

[1] M. L. Gorodetsky, A. A. Savchenkov, and V. S. Ilchenko, "Ultimate Q of optical microsphere resonators", Opt. Lett., 21, pp.453-455 (1996)

[2] D. K. Armani, T. J. Kippenberg, S. M. Spillane, and K. J. Vahala "Ultra-high-Q toroid microcavity on a chip"Nature, 421, pp.925-929 (2003)

[3] V. S. Ilchenko, X. S. Yao, and L. Maleki "Microsphere integration in active and passive photonics devices", Proc. SPIE 3930, pp.154-162 (2000)

[4] I. E. Barry, G. W. Ross, P. G. R. Smith, R.W. Eason, and G. Cook, "Microstructuring of lithium niobate using differential etch-rate between inverted and non-inverted ferroelectric domains", Mat. Lett.,37, pp.246-254 (1998)

[5] C. L. Sones, A. C. Muir, Y. J. Ying, S. Mailis, R. W. Eason, T. Jungk, A. Hoffmann, and E. Soergel, "Precision nanoscale domain engineering of lithium niobate via UV laser induced inhibition of poling” Appl. Phys. Lett. 92, 072905 (2008)

[6] R. Lipowsky, "Surface induced disorder and surface melting" Springer Proc. in Physics, Vol. 50 Springer Verlag Berlin, Heidelberg, pp.158$166(1990)$ 